\title{
Pharmacological Alteration of Cellular Mechanical Properties in Pulmonary Arterial Smooth Muscle Cells of Idiopathic Pulmonary Arterial Hypertension
}

\author{
Shinichi Katsuragi ${ }^{a}$, b, d, Nao Tatsumi ${ }^{\text {c, d }}$, Mizuki Matsumoto ${ }^{c, d}$, Jun Narita ${ }^{\text {a }}$, Ryo Ishiia \\ Hidehiro Suginobe ${ }^{\mathrm{a}}$, Hirofumi Tsuru ${ }^{\mathrm{a}}$, Renjie Wang ${ }^{\mathrm{a}}$, Shigetoyo Kogaki ${ }^{\mathrm{a}}$, , Ryosuke Tanaka ${ }^{\mathrm{c}}$, \\ Keiichi Ozono ${ }^{\text {a }}$, Takaharu Okajima ${ }^{\text {c, e }}$, Hidekazu Ishida ${ }^{\text {a, e }}$ (i)
}

\begin{abstract}
Background: Idiopathic pulmonary arterial hypertension (IPAH) is a progressive disease caused by vascular remodeling of the pulmonary arteries with elevated pulmonary vascular resistance. Recently, various pulmonary vasodilator drugs have become available in the clinical field, and have dramatically ameliorated the prognosis of IPAH. However, little is known about how the mechanical properties of pulmonary arterial smooth muscle cells (PASMCs) are altered under drug supplementation.
\end{abstract}

Methods: Atomic force microscopy (AFM) was used to investigate the mechanical properties of PASMCs derived from a patient with IPAH (PAH-PASMCs) and a healthy control (N-PASMCs) which received the supplementation of clinically used drugs for IPAH: sildenafil, macitentan, and riociguat.

Results: PASMCs derived from PAH-PASMCs were stiffer than those derived from N-PASMCs. With sildenafil treatment, the apparent Young's modulus $\left(E_{0}\right)$ of cells significantly decreased in PAHPASMCs but remained unchanged in N-PASMCs. The decrease in $E_{0}$ of PAH-PASMCs was also observed in macitentan and riociguat treatment. The stress relaxation AFM revealed that the decrease in $E_{0}$ of PAH-PASMCs resulted from a decrease in the cell elastic modulus and/or increase in cell fluidity. The combination treatment of maci-

Manuscript submitted May 18, 2021, accepted June 18, 2021

Published online July 9, 2021

${ }^{a}$ Department of Pediatrics, Osaka University Graduate School of Medicine, Osaka, Japan

${ }^{b}$ Department of Pediatrics and Neonatology, Osaka General Medical Center, Osaka, Japan

${ }^{\mathrm{c}}$ Graduate School of Information Science and Technology, Hokkaido University, Sapporo, Japan

${ }^{\mathrm{d}}$ These authors contributed equally to this work.

e Corresponding Authors: Hidekazu Ishida, Department of Pediatrics, Osaka University Graduate School of Medicine, 2-2 Yamadaoka, Suita, Osaka 5650871, Japan. Email: hideishi@ped.med.osaka-u.ac.jp; Takaharu Okajima, Graduate School of Information Science and Technology, Hokkaido University, N14 W9 Kita-ku, Sapporo 060-0814, Japan.

Email: okajima@ist.hokudai.ac.jp

doi: https://doi.org/10.14740/cr1282 tentan and riociguat showed an additive effect on cell mechanical properties, implying that this clinically accepted combination therapy for IPAH influences the intracellular mechanical components.

Conclusions: Pulmonary vasodilator drugs affect the mechanical properties of PAH-PASMCs, and there exists a mechanical effect of combination treatment on PAH-PASMCs.

Keywords: Pulmonary arterial hypertension; Atomic force microscopy; Smooth muscle cells; Cellular mechanical properties; Elasticity; Fluidity

\section{Introduction}

Idiopathic pulmonary arterial hypertension (IPAH) is a relatively rare, progressive disease with elevated pulmonary vascular resistance (PVR), which leads to right heart failure and eventually death [1]. It is associated with the narrowing of pulmonary arteries, often caused by the constriction and remodeling of the small vessels, and is hemodynamically defined as an elevated mean pulmonary arterial pressure $>20 \mathrm{~mm} \mathrm{Hg}$, elevated PVR $\geq 3$ Wood units, and pulmonary arterial wedge pressure $\leq 15 \mathrm{~mm} \mathrm{Hg}$ [2]. The main target of drug therapy against IPAH has been and still is the reduction of pulmonary arterial pressure by inducing the relaxation of the pulmonary arteries. Despite the recent development of new drugs and multiple combination therapies, the prognosis of IPAH is poor, with a 5-year survival rate of $70 \%$ [3-5]. Therefore, the development of novel therapeutic strategies is urgently required.

The mechanical measurement of pulmonary arterial smooth muscle cells (PASMCs) is important to elucidate how pulmonary arteries cause mechanical changes at the cellular level. Atomic force microscopy (AFM) is a powerful tool for investigating the mechanical properties of living cells [6]. Using AFM, Nakamura et al reported that PASMCs derived from patients with IPAH and normal PASMCs showed different stiffness after nitric oxide supplementation [7]. However, it is still unknown how the mechanical properties, including stiffness and fluidity of PASMCs, are affected by the treatment of pulmonary vasodilator drugs used in clinical practice. Here, 
N-PASMCS

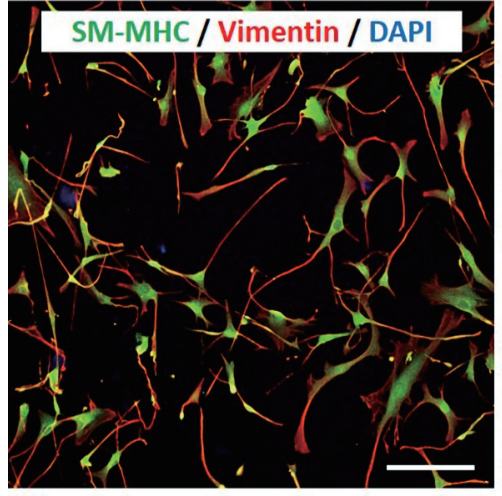

PAH-PASMCS

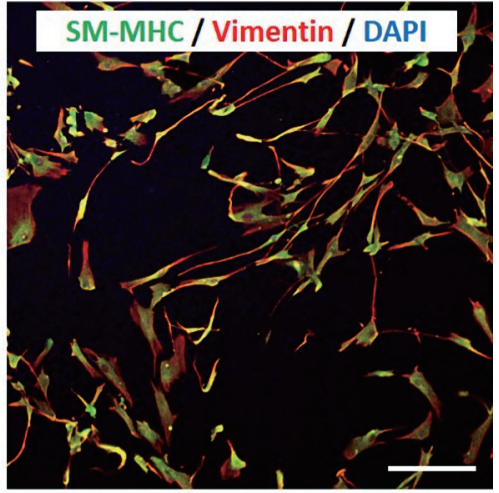

Figure 1. Morphological and immunocytochemical assessments of pulmonary arterial smooth muscle cells (PASMCs) obtained from a healthy control (N-PASMCs) and an idiopathic pulmonary hypertension patient (PAH-PASMCs). Immunocytochemistry of smooth muscle myosin heavy chain (SM-MHC) and vimentin revealed that almost all cells were SM-MHC-positive smooth muscle cells, and their morphological features were similar between N-PASMCs and PAH-PASMCs. Scale bar: $100 \mu \mathrm{m}$. DAPI: 4', 6-diamidino-2-phenylindole.

we investigated the mechanical properties of PASMCs derived from a patient with IPAH (PAH-PASMCs) in response to clinically used pulmonary vasodilators, such as sildenafil, riociguat, and macitentan, by AFM. We found that these drugs caused softening of PAH-PASMCs, in which the elastic components of cells were reduced prior to the viscous (fluidic) component. Furthermore, we found that the combination of macitentan and riociguat had an additive effect on the mechanical properties of PAH-PASMCs. The results indicated that the AFM measurement provides a quantitative assessment of pulmonary vasodilators in PAH-PASMCs, which may be useful for drug assays.

\section{Materials and Methods}

\section{Harvest and culture of PASMCs}

PASMCs were harvested from a piece of excised pulmonary arterial tissue obtained from a 13-year old male patient with IPAH during lung transplantation. Tunica intima and tunica adventitia, as well as the extracellular matrix, were manually removed from tunica media under a stereomicroscope (SZX10, Olympus, Tokyo, Japan). The sample was minced and incubated in $37^{\circ} \mathrm{C}$ for $15 \mathrm{~min}$ in papain solution, followed by collagenase and elastase digestion. The suspended cells were washed with phosphate buffered saline, centrifuged, and then incubated in a humidified $5 \% \mathrm{CO}_{2}$ atmosphere at $37{ }^{\circ} \mathrm{C}$ with AmnioMax-II complete media (Thermo Fisher Scientific, MA, USA). After the first passage, the cells were cultured and maintained in SmGM-2 (smooth muscle cell growth medium, Lonza, Basel, Switzerland). The purity of PASMCs was examined by immunocytochemistry using anti-smooth muscle myosin heavy chain (SM-MHC) and anti-vimentin (both purchased from Abcam, Cambridge, UK). As a non-disease control, commercially available normal PASMCs were purchased from Lonza, and the cells were cultured in SmGM-2.
Both normal PASMCs (N-PASMCs) and IPAH patient-derived PASMCs (PAH-PASMCs) were used for the experiments between passages six and eight. N-PASMCs and PAH-PASMCs were morphologically similar, and all cells were SM-MHCpositive and vimentin-positive smooth muscle cells (Fig. 1). All experiments were approved by the Clinical Research Ethical Committee of Osaka University (No. 15118). This study was conducted in compliance with the Helsinki Declaration. Both N-PASMCs and PAH-PASMCs were cultured in 35-mm cell culture dishes. The confluency of the cell samples was $90 \%$ in SmGM-2 medium on the day of analysis. Before the AFM experiment, the medium was replaced with a $\mathrm{CO}_{2}$-independent medium.

\section{AFM measurement}

The mechanical properties of N-PASMCs and PAH-PASMCs in untreated and drug-treated conditions were measured using a customized atomic force microscope attached to an upright optical microscope (Eclipse FN1, Nikon, Tokyo, Japan), as reported previously (Fig. 2a) [8, 9]. The deflection of the rectangular cantilever (BioLever mini, BL-AC40TS-C2, Olympus, Tokyo, Japan) was detected through a water-immersed objective lens (CFI Plan fluor $10 \times \mathrm{W}$, Nikon). The loading force was determined using Hooke's law by multiplying the cantilever deflection with the spring constant, calibrated using a thermal fluctuation method. Force measurements were performed around the cell center to eliminate the effect of cell thickness on cell mechanical properties. The $E_{0}$ for each force curve (Fig. 2b) was estimated using the Sneddon's model as follows [6]:

$$
F=\frac{2 \tan \theta}{\pi} \frac{E_{0}}{1-v^{2}} \delta^{2},
$$

where $F$ is the loading force, $\delta$ is the indentation depth, $\theta$ is the half-opening angle of the AFM conical indenter of $17.5^{\circ}$, and $v$ is the Poisson's ratio, which was assumed to be 0.5 . The $E_{0}$ 

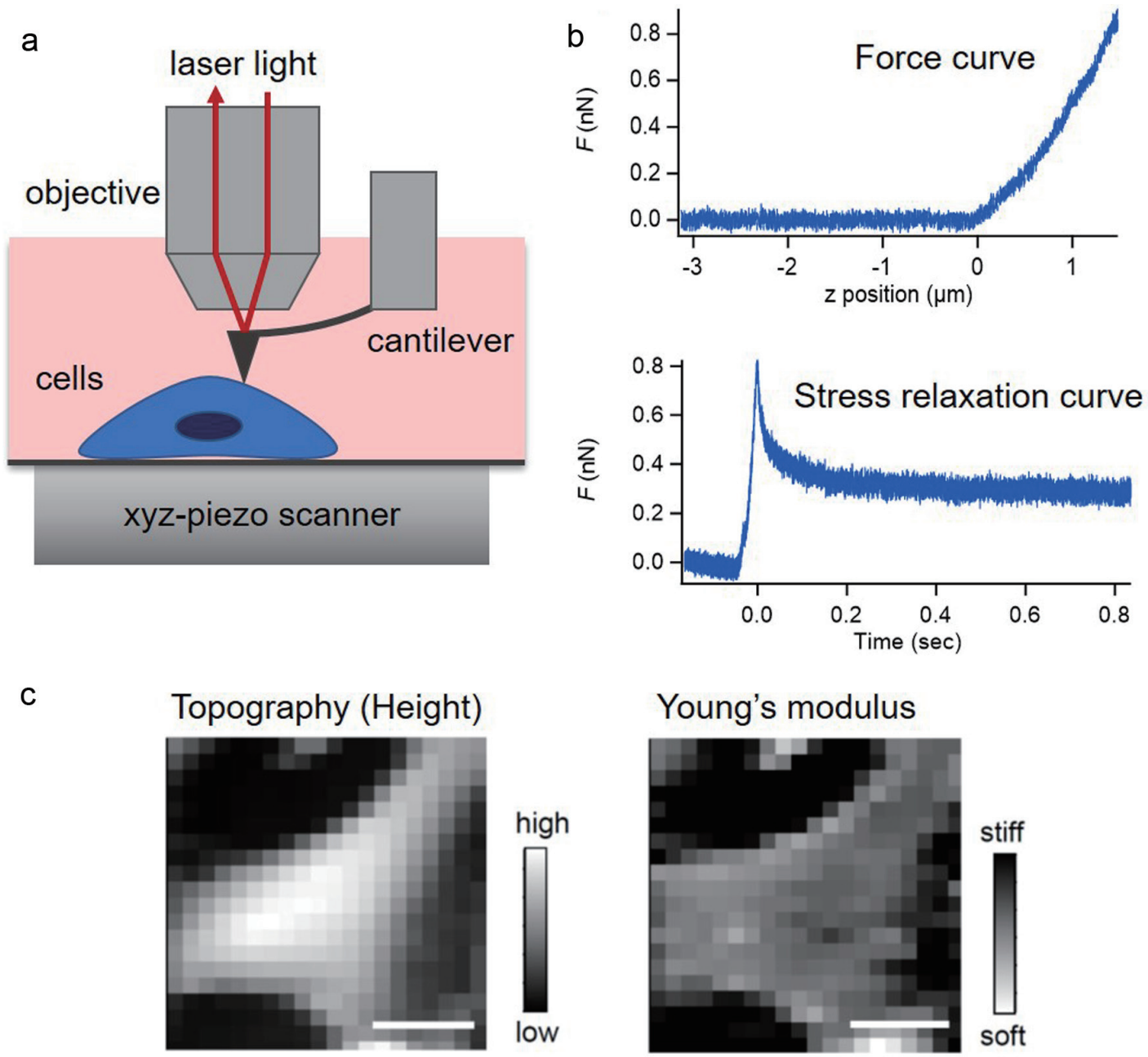

Figure 2. Atomic force microscopy (AFM) measurement of pulmonary arterial smooth muscle cells (PASMCs). (a) Schematic of AFM measurement for PASMCs. (b) Typical force curve (upper panel) and stress relaxation curve (lower panel) of PASMCs in AFM measurement. (c) An example of topographic and the apparent Young's modulus images of PASMC obtained in AFM force curve measurement. Scale bar: $30 \mu \mathrm{m}$.

values measured at different locations around the cell center were averaged. We estimated the ratio of $E_{0}$ of drug-treated cells to that of untreated cells, $E_{\mathrm{c}}$, to minimize day-to-day variability in cell mechanical measurements.

For stress relaxation AFM measurement, the cantilever base displacement approached the cell sample at a constant speed and was then held constant while the cells were indented with a loading force, and the relaxation behavior during the indentation was measured (Fig. 2b) [6]. In this case, the $E_{0}$ in the equation was replaced with the relaxation modulus of $E(t)$, approximately following the power-law rheology expressed as $E(t)=E_{1} t^{-\alpha}$, where $t$ is the time, $E_{1}$ is the elastic modulus at 1 $\mathrm{s}$, and $\alpha$ is the power-law exponent between 0 for a solid and 1 for a liquid $[6,10-12]$. Previous studies have shown that the modulus and the power-law exponent exhibited log-normal and normal distributions, respectively [13].

PASMCs were treated with three commercially available drugs for PAH: sildenafil, riociguat, and macitentan (all purchased from Cayman Chemical, MI, USA). All the drugs were dissolved in dimethyl sulfoxide (DMSO). The cell samples were incubated for $24 \mathrm{~h}$ after the drug supplementation, and then the media were replaced with $\mathrm{CO}_{2}$-independent media before the AFM experiment.

\section{Statistical analysis}

For data analysis, Student's $t$-test and one-way analysis of variance (ANOVA) followed by Tukey's test were used to determine $\mathrm{P}$ values, as indicated in the figures. Statistical significance was set at $\mathrm{P}<0.05$. Error bars indicate standard deviations.

\section{Results}

We first confirmed the feasibility and reliability of AFM for examination of the PASMC samples used in this study. Fig- 
a

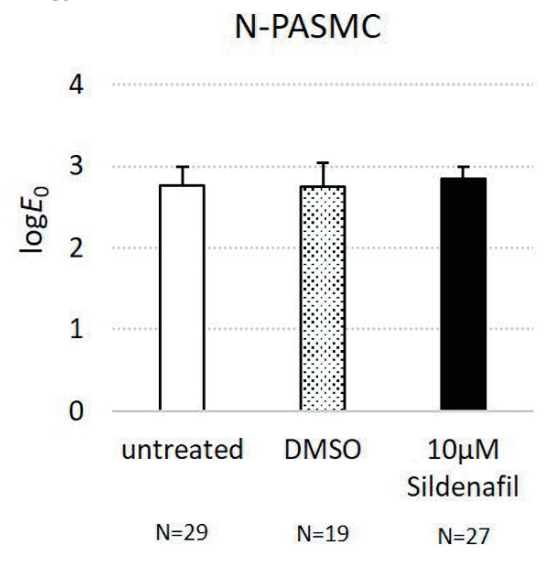

b

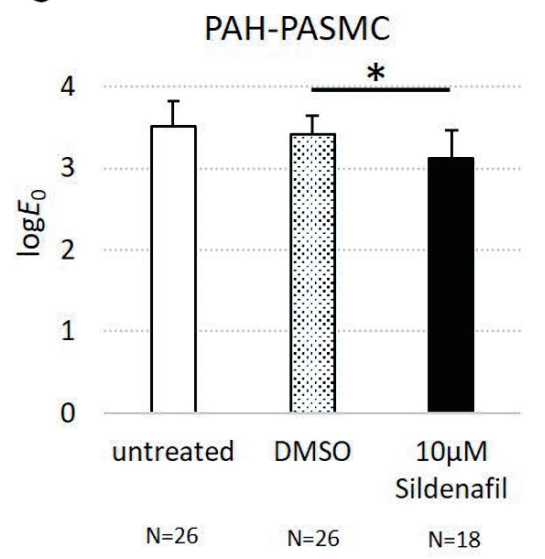

Figure 3. The apparent Young's modulus of pulmonary arterial smooth muscle cells (PASMCs) derived from healthy controls (N-PASMCs) and PASCMCs derived from a patient with idiopathic pulmonary arterial hypertension (PAH-PASMCs) in response to sildenafil treatment. (a) The $\log E_{0}$, of N-PASMCs in untreated, DMSO, and sildenafil-treated conditions. (b) The $\log E_{0}$ of PAHPASMCs in untreated, DMSO, and sildenafil-treated conditions. DMSO: dimethylsulfoxide.

ure 2c shows representative AFM mapping images of the cell height (topography) and the $E_{0}$ of PASMCs. Single cells were clearly captured in both the images, showing that AFM was capable of measuring the mechanical properties of the cell without substantial damage at the subcellular level.

To investigate the mechanical effect of clinically available drugs on PASMCs, we measured the cell stiffness, that is, the apparent Young's modulus, $E_{0}$, of N-PASMC and PAH-PASMC cells in response to sildenafil, a phosphodiesterase 5 inhibitor, which increases intracellular cyclic guanosine monophosphate (cGMP) concentration, leading to cellular relaxation. We observed that PAH-PASMCs significantly decreased $E_{0}$ when treated with sildenafil, whereas in case of N-PASMCs, no significant decrease was observed (Fig. 3). These results suggested that PAH-PASMCs are stiffer than N-PASMCs, and sildenafil can significantly soften PAH-PASMCs.

Next, we investigated whether the cellular mechanical properties of PAH-PASMCs were affected by treatment with macitentan, an endothelin receptor antagonist. We observed that macitentan concentrations more than $10 \mu \mathrm{M}$ caused a significant reduction in the $E_{0}$ of PAH-PASMCs (Fig. 4a). These results clearly demonstrated that both cGMP enhancement and endothelin signal blockade could reduce the $E_{0}$ of PAHPASMCs. In contrast, the stress relaxation AFM experiment showed no significant difference in $E_{1}$ (relaxation modulus at $1 \mathrm{~s}$ ), and $\alpha$ (power-law exponent of the relaxation modulus as a function of time) was observed at macitentan concentration of $10 \mu \mathrm{M}$. As the concentration of macitentan was further increased to more than $15 \mu \mathrm{M}$, we observed a significant reduction in $E_{1}$ and an increase in $\alpha$, thus suggesting cell softening and fluidization (Fig. 4b, c). These results indicated that PAHPASMCs in vitro changed cellular rheological properties at a threshold concentration of macitentan between $10 \mu \mathrm{M}$ and 15 $\mu \mathrm{M}$.

Riociguat is a relatively new clinically available drug that induces intracellular cGMP production by stimulating soluble guanylate cyclase. Thus, riociguat can induce cGMP produc- tion in a nitric oxide-independent manner. In case of PAHPASMCs treated with $50 \mu \mathrm{M}$ riociguat, a significant reduction in $E_{0}$ (Fig. $4 \mathrm{~d}$ ) was observed, while $E_{1}$ and $\alpha$ remained unchanged (Fig. 4e, f), which was similar to the behavior observed with $10 \mu \mathrm{M}$ macitentan. We also observed a decrease in $E_{1}$ (Fig. 4f) and an increase in $\alpha$ (Fig. 4e) as the concentration of riociguat was further increased to $62 \mu \mathrm{M}$. The results indicated that PAH-PASMCs changed $\alpha$ and $E_{1}$ at a threshold concentration of riociguat between $50 \mu \mathrm{M}$ and $62 \mu \mathrm{M}$.

We investigated the mechanical properties of PAHPASMCs treated with the combination of macitentan and riociguat, which is widely used in the clinics for the treatment of IPAH [1]. As shown in Figure 4g, compared to untreated cells, PAH-PASMCs exhibited no significant change at riociguat concentration of $25 \mu \mathrm{M}$. Upon further addition of $10 \mu \mathrm{M}$ of macitentan to the $25 \mu \mathrm{M}$ riociguat-treated PAH-PASMCs, we observed that not only $E_{0}$, but $E_{1}$ was also significantly decreased, and a decrease in stiffness initially occurred prior to cell fluidization (Fig. 4h, i). This result indicated that the combination of riociguat and macitentan effectively contributed to the reduction in stiffness of PAH-PASMCs. Taken together, sildenafil, macitentan, and riociguat caused the softening of PAH-PASMCs, and the combination therapy additively affected the stiffness of PAH-PASMCs.

\section{Discussion}

IPAH is a progressive obstinate disease characterized by the elevation of PVR due to the contraction and remodeling of the pulmonary arteries. The development of pulmonary vasodilator drugs in the past two decades has dramatically improved the mortality and morbidity of patients with IPAH [1].These drugs can relax the constricting pulmonary arterial smooth muscles. However, the mechanical properties of PASMCs at the cellular level in response to drugs used for the treatment of IPAH are unclear. In this study, we used AFM to investigate 
a

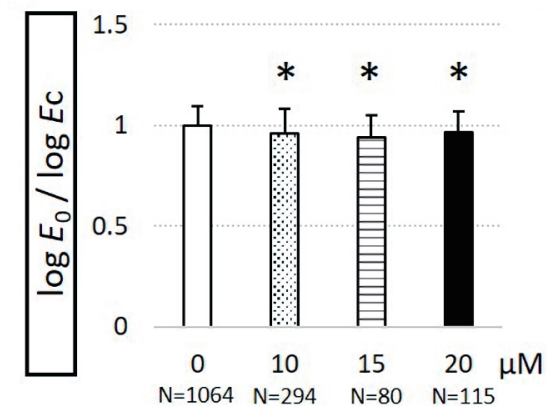

b

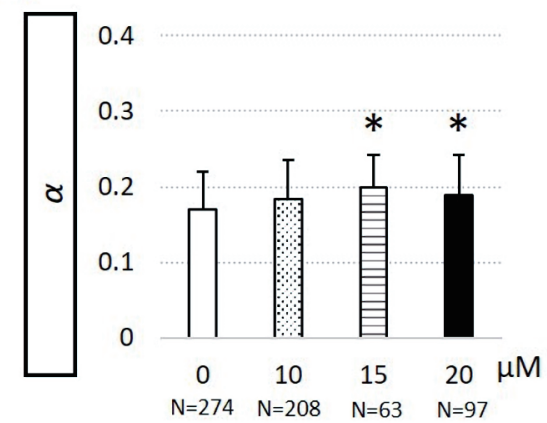

c

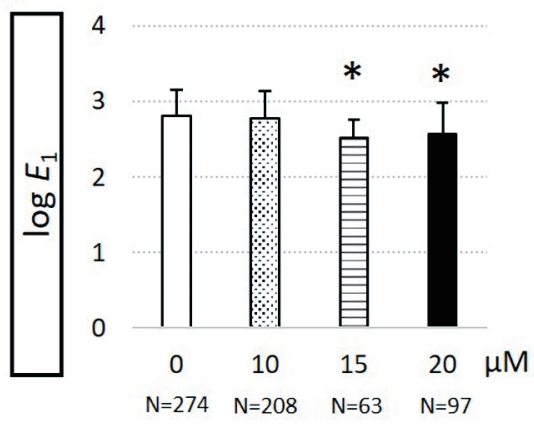

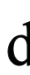
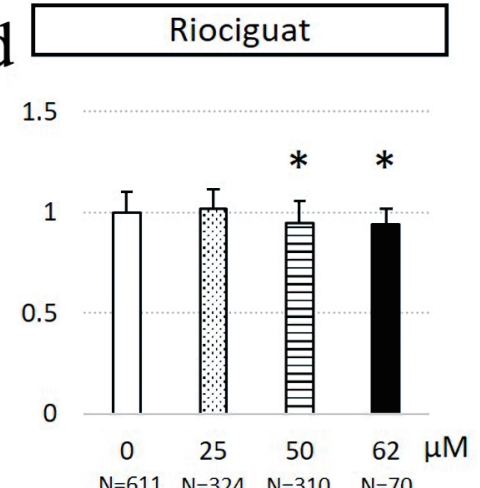

e

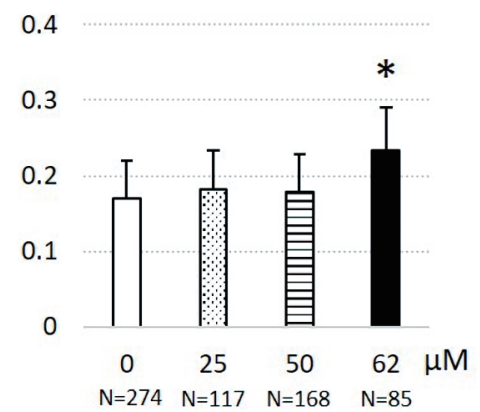

f

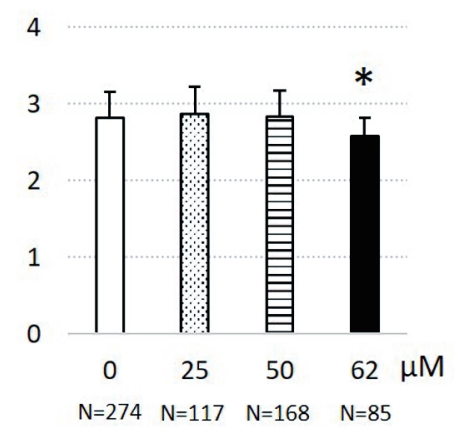

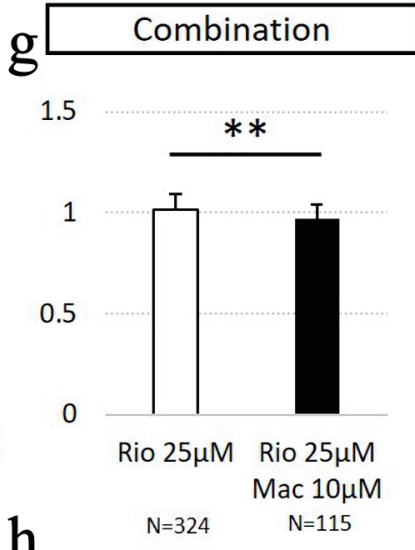

0.4

0.3

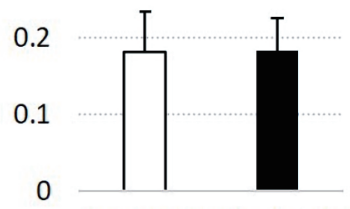

Rio $25 \mu \mathrm{M}$ Rio $25 \mu \mathrm{M}$

Mac $10 \mu \mathrm{M}$

i

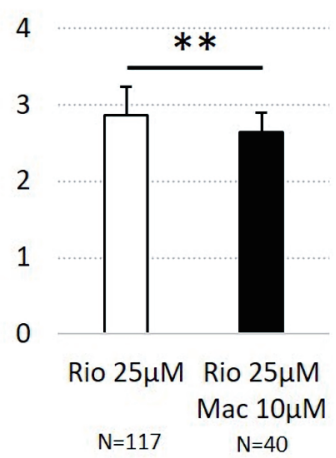

Figure 4. Mechanical properties of pulmonary arterial smooth muscle cells (PASCMCs) derived from a patient with idiopathic pulmonary arterial hypertension (PAH-PASMCs) in response to macitentan and riociguat treatment. (a) The apparent Young's modulus normalized with that of untreated cells, $\log E_{0} / \log E_{\mathrm{c}}$ of PAH-PASMCs in response to macitentan treatment. (b) $\alpha$ of macitentan-treated PAH-PASMCs. (c) The $\log E_{1}$ of macitentan-treated PAH-PASMCs. (d) The $\log E_{0} / \log E_{\mathrm{c}}$ of riociguat-treated PAH-PASMCs. (e) $\alpha$ of riociguat-treated PAH-PASMCs. (f) The $\log E_{1}$ of riociguat-treated PAH-PASMCs. (g) The $\log E_{0} / \log E_{\mathrm{c}}$ of PAH-PASMCs with combination treatment. (h) $\alpha$ of PAH-PASMCs with combination treatment. (i) The log $E_{1}$ of PAH-PASMCs with combination treatment. ${ }^{*} \mathrm{P}<0.05$ vs. DMSO-treated PASMCs by ANOVA. ${ }^{* *} \mathrm{P}<0.05$ by Student's $t$-test. DMSO: dimethylsulfoxide; ANOVA: analysis of variance.

the alteration of the mechanical properties of PASMCs derived from an IPAH patient.

First, we demonstrated that the cellular stiffness of PAHPASMCs was significantly decreased after sildenafil treatment, whereas that of N-PASMCs was not affected. We also revealed that macitentan and riociguat treatment reduced $E_{0}$, and $E_{1}$, and increased $\alpha$. It should be noted that such a negative correlation between the elastic modulus and the power- law exponent has been commonly observed in various types of in vitro single cells $[10,13]$. These results indicated that both endothelin signal blockade and cGMP stimulation affect the cellular stiffness and fluidity of PAH-PASMCs, which may change from a mechanical state to other states through normal mechanical pathways.

Previous studies have reported that both the nitric oxidecGMP pathway and endothelin signaling pathway alter the in- 
tracellular $\mathrm{Ca}^{2+}$ concentration and sensitivity $[14,15]$. Therefore, each drug supplementation can exert the relaxation of smooth muscle cells. It is noteworthy in our study that AFM could clearly delineate the decrease in stiffness and increase in fluidity of PASMCs in response to drug treatment. These alterations in the cellular mechanical properties of PAHPASMCs, induced by pulmonary vasodilation drugs, could be a key aspect of pulmonary artery relaxation for the treatment of patients with IPAH. Intriguingly, we observed that the $E_{0}$ of PAH-PASMCs was higher than that of N-PASMCs, and we did not observe softening of N-PASMCs in response to sildenafil treatment. These results indicate that the cellular stiffness of PASMCs is elevated in IPAH, and such cellular mechanical properties are maintained even under primary culture in vitro. Therefore, we speculated that the clinical effects of pulmonary vasodilators may be observed by AFM using PAH-PASMCs in an in vitro assay system. However, in this study, we analyzed the PASMCs derived from only one patient. This is the main limitation of this study, and further investigation is required using other controls and PAH patientderived PASMCs.

Finally, we analyzed the effect of combination therapy, which is widely recommended in the clinical treatment of IPAH $[16,17]$. We found that the dual supplementation of macitentan and riociguat could reduce the threshold of $E_{1}$ in PAH-PASMCs at a lower concentration of both the drugs. In contrast, the fluidity did not change with the combination of low concentrations of macitentan and riociguat. These results indicate that the cellular stiffness of PAH-PASMCs is additively affected by cGMP signaling and endothelin signal blockade, which corresponds to the results of human studies and animal experiments $[16,18]$.

The main limitation of this study is the small sample size. We analyzed only one patient's PASMCs. Therefore, studies with a larger sample size are required. Another limitation is the discrepancy between clinically used and in vitro concentrations of the supplemented drugs. We determined the concentrations of sildenafil and riociguat by our previous experiments wherein we measured cGMP concentration in PASMCs after treatment with sildenafil and riociguat in vitro [19]. We confirmed that $5-10 \mu \mathrm{M}$ sildenafil could effectively induce intracellular cGMP in PASMCs. Similarly, we also confirmed that $50-100 \mu \mathrm{M}$ riociguat could significantly increase the intracellular cGMP concentration of PASMCs. Regarding macitentan, a previous study demonstrated that $10 \mu \mathrm{M}$ macitentan could effectively inhibit inositol phosphate 1 accumulation induced by ET-1 stimulation [20]. According to the clinical pharmacokinetics, the serum concentration of these drugs in the patients under the clinically used dosage was $0.5-2 \mu \mathrm{M}$. However, there are no data on their concentrations in lung tissues or pulmonary smooth muscle cells in vivo. We think that this is a limitation of in vitro studies using cultured cells.

In summary, we investigated the mechanical properties of PASMCs in terms of $E_{0}$, corresponding to cell stiffness, $E_{1}$, corresponding to the time-dependent elastic modulus, and $\alpha$, corresponding to cell fluidity, using AFM. AFM measurements revealed that PAH drug treatments caused significant softening of PAH-PASMCs. The combination treatment additively affected the mechanical properties of PAH-PASMCs. These results indicate the potential value of AFM for in vitro assessment of the pathological mechanisms of PAH and novel drug screening.

\section{Acknowledgments}

None to declare.

\section{Financial Disclosure}

This work was supported by grants from the Ministry of Education, Science, Sports, and Culture of Japan (No. 16K10065 and 19K08276).

\section{Conflict of Interest}

None to declare.

\section{Informed Consent}

All the patients who were included in this study gave their informed consent.

\section{Author Contributions}

SK, HS, HT and RW harvested and cultured PASMCs. NT, $\mathrm{MM}$ and RT measured the cells using AFM. JN, RI and SK managed the patient's cell preparation. HI and TO wrote the manuscript and managed all experiments. HI, SK and KO discussed about the biological feasibility and meaning of the results. All authors approved the final version of the manuscript.

\section{Data Availability}

The data supporting the findings of this study are available from the corresponding author upon reasonable request.

\section{References}

1. Galie N, Humbert M, Vachiery JL, Gibbs S, Lang I, Torbicki A, Simonneau G, et al. 2015 ESC/ERS Guidelines for the diagnosis and treatment of pulmonary hypertension: the joint task force for the diagnosis and treatment of pulmonary hypertension of the European Society of Cardiology (ESC) and the European Respiratory Society (ERS): endorsed by: Association for European Paediatric and Congenital Cardiology (AEPC), International Society for Heart and Lung Transplantation (ISHLT). Eur Heart J. 2016;37(1):67-119.

2. Simonneau G, Montani D, Celermajer DS, Denton CP, Gatzoulis MA, Krowka M, Williams PG, et al. Haemody- 
namic definitions and updated clinical classification of pulmonary hypertension. Eur Respir J. 2019;53(1):1801913.

3. Galie N, Ghofrani HA, Torbicki A, Barst RJ, Rubin LJ, Badesch D, Fleming T, et al. Sildenafil citrate therapy for pulmonary arterial hypertension. N Engl J Med. 2005; 353(20):2148-2157.

4. Pulido T, Adzerikho I, Channick RN, Delcroix M, Galie N, Ghofrani HA, Jansa P, et al. Macitentan and morbidity and mortality in pulmonary arterial hypertension. N Engl J Med. 2013;369(9):809-818.

5. Ghofrani HA, Galie N, Grimminger F, Grunig E, Humbert M, Jing ZC, Keogh AM, et al. Riociguat for the treatment of pulmonary arterial hypertension. N Engl J Med. 2013;369(4):330-340.

6. Efremov YM, Okajima T, Raman A. Measuring viscoelasticity of soft biological samples using atomic force microscopy. Soft Matter. 2020;16(1):64-81.

7. Nakamura K, Shimizu J, Kataoka N, Hashimoto K, Ikeda T, Fujio H, Ohta-Ogo K, et al. Altered nano/micro-order elasticity of pulmonary artery smooth muscle cells of patients with idiopathic pulmonary arterial hypertension. Int J Cardiol. 2010;140(1):102-107.

8. Fujii Y, Ochi Y, Tuchiya M, Kajita M, Fujita Y, Ishimoto Y, Okajima T. Spontaneous spatial correlation of elastic modulus in jammed epithelial monolayers observed by AFM. Biophys J. 2019;116(6):1152-1158.

9. Tanaka R, Sawano M, Fujii Y, Kuribayashi-Shigetomi K, Subagyo A, Sueoka K, Okajima T. Relationship between rheological properties and actin filaments of single cells investigated by atomic force microscopy. Jpn J Appl Phys. 2020;59:SN1010.

10. Kollmannsberger P, Fabry B. Linear and nonlinear rheology of living cells. Annual Review of Materials Research. 2011;41:75-97.

11. Takahashi R, Okajima T. Comparison between power-law rheological parameters of living cells in frequency and time domains measured by atomic force microscopy. Jpn J Appl
Phys. 2016;56:08NB22.

12. Hiratsuka S, Mizutani Y, Toda A, Fukushima N, Kawahara $\mathrm{K}$, Tokumoto H, Okajima T. Power-law stress and creep relaxations of single cells measured by colloidal probe atomic force microscopy. Jpn J Appl Phys. 2009;48:08JB17.

13. Cai P, Mizutani Y, Tsuchiya M, Maloney JM, Fabry B, Van Vliet KJ, Okajima T. Quantifying cell-to-cell variation in power-law rheology. Biophys J. 2013;105(5):1093-1102.

14. Carvajal JA, Germain AM, Huidobro-Toro JP, Weiner CP. Molecular mechanism of cGMP-mediated smooth muscle relaxation. J Cell Physiol. 2000;184(3):409-420.

15. Wynne BM, Chiao CW, Webb RC. Vascular smooth muscle cell signaling mechanisms for contraction to angiotensin II and endothelin-1. J Am Soc Hypertens. 2009;3(2):84-95.

16. Galie N, Barbera JA, Frost AE, Ghofrani HA, Hoeper MM, McLaughlin VV, Peacock AJ, et al. Initial Use of Ambrisentan plus Tadalafil in Pulmonary Arterial Hypertension. N Engl J Med. 2015;373(9):834-844.

17. Sitbon O, Sattler C, Bertoletti L, Savale L, Cottin V, Jais $\mathrm{X}$, De Groote $\mathrm{P}$, et al. Initial dual oral combination therapy in pulmonary arterial hypertension. Eur Respir J. 2016;47(6):1727-1736.

18. Lachant DJ, Meoli DF, Haight D, Staicu S, Akers S, Glickman S, Ambrosini R, et al. Combination therapy improves vascular volume in female rats with pulmonary hypertension. Am J Physiol Lung Cell Mol Physiol. 2019;317(4):L445-L455.

19. Nawa N, Ishida H, Katsuragi S, Baden H, Takahashi K, Higeno R, Torigoe F, et al. Constitutively active form of natriuretic peptide receptor 2 ameliorates experimental pulmonary arterial hypertension. Mol Ther Methods Clin Dev. 2016;3:16044.

20. Gatfield J, Mueller Grandjean C, Sasse T, Clozel M, Nayler O. Slow receptor dissociation kinetics differentiate macitentan from other endothelin receptor antagonists in pulmonary arterial smooth muscle cells. PLoS One. 2012;7(10):e47662. 